\title{
O AtoM COMO RECURSO DESCRITIVO WEB DE REPRESENTAÇÃO DE ARQUIVOS PÚBLICOS: BRASIL E COLÔMBIA
}

\author{
AtoM as descriptive Web resource for representation of Public Archives: Brazil and Colombia
}

\section{Maria José Vicentini Jorente (1), Talita Cristina da Silva (1), Aura Marcela Mesa (2), Mateus Henrique da Silva}

(1) UNESP - Universidade Estadual Paulista "Júlio de Mesquita Filho”, mjjorente@ marilia.unesp.br, talita_arquivo@hotmail.com, mattituh@gmail.com (2) Universidad de Antioquia - Escuela Interamericana de Bibliotecología, ammp8825@gmail.com.

\begin{abstract}
Resumo
Este trabalho averigua como a literatura da área discute o processo de descrição arquivística no Brasil e na Colômbia. O objetivo geral é investigar como a descrição arquivística e o AtoM têm sido abordados nas instituições de Arquivos Públicos latino americanos. No Brasil o AtoM é experimentado, enquanto na Colômbia não se verifica a sua disseminação. Como objetivos específicos comparam-se as literaturas sobre dois softwares colombianos - Mercurio e ENKI JANIUM - e o internacional AtoM por meio de pesquisa bibliográfica. Este trabalho justifica-se pela importância do processo de descrição arquivística na socialização, representação e acesso à informação de instituições e documentos de arquivo e também pela necessidade de promoção e de disseminação dos padrões internacionais e nacionais de descrição arquivística e do sistema AtoM. Os resultados esperados são relacionados aos processos de descrição utilizados no Brasil e na Colômbia e à aplicação das normas internacionais em ambos os países. Conclui-se que o Design do AtoM, corresponde às necessidades de acesso a informação em um ambiente digital na Web, bem como à sua descrição, ao produtor, à instituição custodiadora e a função arquivística.
\end{abstract}

Palavras-chave: Descrição; Descrição Arquivística; AtoM; ICA-AtoM; Design da Informação.

\section{Normalização e acesso a informações de Arquivo}

Considerando o cenário atual de desenvolvimento e uso exponencial das mídias digitais e a realidade de instituições de Arquivo - na qual os indivíduos que procuram os diversos ambientes de informação atuam de forma proativa na busca pelo acesso às informações, maior velocidade e relevância nas respostas das buscas - encontra-se a problemática deste artigo: a necessidade de padrões e sistemas normalizados em Arquivo para que o intercâmbio de informações seja possível e que o acesso aos documentos custodiados pelas instituições seja efetivo.

\begin{abstract}
This study investigates how the literature of the area discusses the archival description process in Brazil and Colombia. The general objective is to reveal how Latin American Public Archival institutions have addressed archival description and AtoM. In Brazil, AtoM is in use while in Colombia its dissemination is not verified. As specific objectives, this study compares the literatures on two Colombian systems - Mercurio and ENKI JANIUM - and the international AtoM in the literature. The study is justified by the importance that archival description plays on the socialization process, representation and access to information of institutions and archival documents, as well as the need to promote and disseminate national and international standards for archival description and the AtoM system. The expected results are related to the description processes used in Brazil and in Colombia, and the application of international standards in both countries. It is concluded that AtoM's design meets the needs for information access in a digital environment on the Web, as well as its description, to the producer, to the custodial institution and to the archival function.
\end{abstract}

Keywords: Description; Archivistic; Description; AtoM; ICA-AtoM; Information Design.

Ao investigar a normalização documental na Arquivologia, percebe-se que a primeira atividade arquivística normalizada foi a Descrição Arquivística. Segundo Llanes Padrón (2011), o processo de normalizar a atividade de descrição arquivística começou com os norte-americanos e canadenses, mas tornou-se um procedimento internacional quando o Conselho Internacional de Arquivos (ICA) tomou para si a missão de construir normas internacionais norteadoras deste processo. A normalização, por sua vez, torna-se importante porque "facilita a realização do processo de descrição, e ainda permite que os instrumentos de pesquisa possam ser utilizados/compreendidos universalmente" (Carpes, Flores, 2014, p,71). 
Segundo o Dicionário de Terminologia Arquivística: descrição é um "conjunto de procedimentos que leva em conta os elementos formais e de conteúdo dos documentos para elaboração de instrumentos de pesquisa" (Arquivo Nacional, 2004, p. 59).

No processo de descrição, os elementos do documento são identificados e posteriormente compilados em um instrumento de pesquisa. Os chamados instrumentos de pesquisa são produtos oriundos das descrições arquivísticas. Por meio destes instrumentos, os indivíduos que buscam informações em arquivos poderão encontrar o documento ou o grupo de documentos que intenciona pesquisar. Guias, inventários, catálogos e índices são exemplos destes instrumentos de pesquisa. O guia é construído a partir das descrições quando o nível é o fundo ou a coleção, enquanto o catálogo se constrói quando se descrevem os níveis intermediários (grupo, classe, série, seção) da classificação. O inventário, por sua vez, se constitui quando a descrição é feita com todos os itens ou unidades documentais do acervo, e os índices podem ser temáticos, alfabéticos ou numéricos. Todos estes instrumentos são gerados a partir do processo de descrição.

Foi para este processo de descrição que se iniciou a criação das normas internacionais de descrição em arquivos e, durante o desenvolvimento desta normalização, foi impulsionado um movimento em diversos países do mundo, no intuito de organizar este processo. (Llanes Padrón, 2011).



Figura 1: Tipos de entidades nos modelos da Austrália, Espanha e Finlândia (Llanes Padrón, 2011, Tradução nossa).

Para realizar a normalização o ICA, onze entidades presentes nas atividades em arquivo são extraídas da literatura internacional da área, as quais mantêm relações entre si, ou seja, um arquivo tem relação orgânica com as diversas entidades presente no arquivo. Ainda quanto às questões de entidade e relação, compreendese que um 'documento' é produzido por um 'produtor' e um 'produtor' produz um documento de acordo com sua 'função'. Outras relações possíveis acontecem quando um documento é produzido em um 'tempo' e está sob a custódia de uma instituição custodiadora.

Percebe-se então por meio destes exemplos que documentos de arquivos são de natureza complexa, pois mantém muitas relações orgânicas intrínsecas e extrínsecas à sua estrutura.

Das entidades identificadas na literatura o ICA promove a normalização da atividade de descrição da entidado documento. Diante disso, o Conselho Internacional de Arquivo (ICA) desenvolveu a primeira norma internacional de descrição a "Norma Geral Internacional de Descrição Arquivística - ISAD (G)"'. Devido à necessidade de padrões internacionais em Arquivo havia uma expectativa por essa normatização pois, como salienta Padrón, a resposta que a padronização obteve foi surpreendente com uma rápida aceitação destes padrões e igualmente rápida aplicação e adaptação destas normas em diversos países. A ISAD (G) foi publicada em 1999, posteriormente, o ICA publica outras três normas internacionais: ISAAR (CPF), ISDIAH, ISDF.

Estas normas internacionais são adaptáveis às especificidades de cada país. Assim, estes países e/ou instituições podem utilizá-las conforme suas necessidades, pois as normas internacionais definem os elementos que devem estar contidos na descrição documental e nas adaptações de cada país devem estar contidas as diretrizes de conteúdo para cada metadado (campo, elemento, área) estabelecido na norma de estrutura.

ICA (conselho internacional de arquivos - CIA)

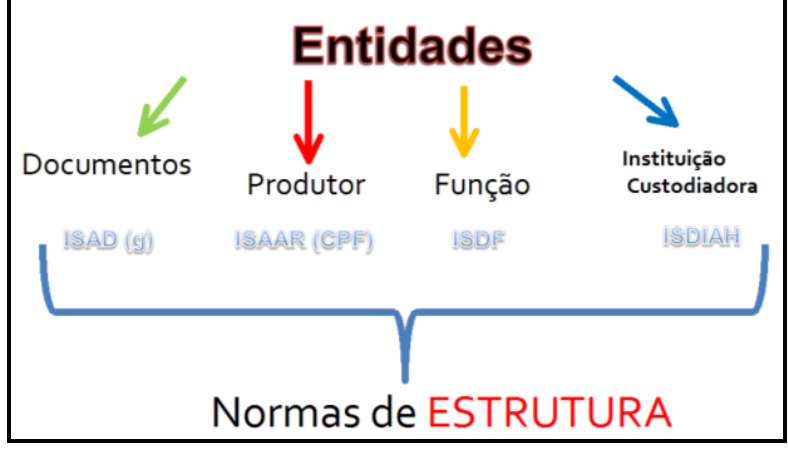

Figura 2: As normas de descrição desenvolvidas pelo ICA.

Como exemplo, podemos citar o que ocorreu com a norma internacional ISAD (G) de 1999. Quando o Arquivo Nacional do Brasil publicou a norma traduzida em 2000, tratava-se apenas de uma tradução da norma original em inglês para o português. Contudo, em 2001, o Conselho Nacional de Arquivos - CONARQ - publicou a NOBRAD, uma adaptação da $\operatorname{ISAD}(\mathrm{G})$, com contribuições da ISAAR (CPF).

Tanto a ISAD (G) quanto a NOBRAD são, portanto, normas de descrição de documentos; a diferença entre elas é que enquanto a primeira define a estrutura dos campos ou elementos de descrição, a segunda define quais informações cada campo deve conter. Ou seja, se na ISAD (G) define-se "Código de referência" como um campo descritivo que deve conter o código do país e da instituição, a NOBRAD define que no campo "Código de referência" o código usado para país será "BR", seguido do nome da instituição. 
Na Colômbia, por outro lado, o Instituto Colombiano de Normas Técnicas e Certificação (ICONTEC) criou em 1997 a Norma Técnica Colombiana NTC 4095 com a colaboração de diferentes entidades - entre elas o Arquivo Geral da Nação da Colômbia (AGN). Esta norma é também uma adaptação da ISAD (G) e está sujeita a ser atualizada permanentemente para responder às necessidades e exigências requeridas. A norma demanda uma "[...] descrição racional e hierarquizada, que seja do geral ao particular" como anuncia o seguinte gráfico (ICONTEC, 1997, p 2, tradução nossa):

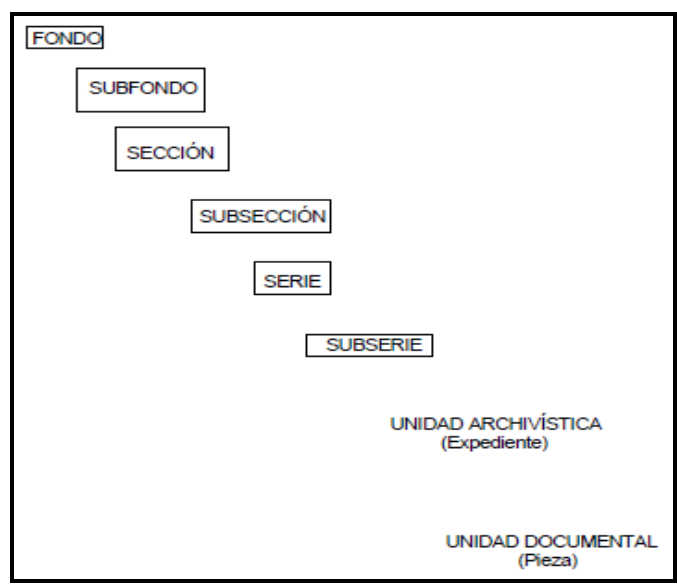

Figura 3: ICONTEC. (Norma Técnica Colombiana 4095)

A ISAAR (CPF) é uma norma de descrição de pessoas, entidades ou famílias. O Brasil não possui uma adaptação desta norma, pois ela apenas foi usada para auxiliar na regulamentação do campo "produtor/acumulador" da NOBRAD. A ISDIAH é uma norma de descrição voltada para a instituição custodiadora, enquanto a ISDF é uma norma de descrição de função. Destaca-se que a ISDIAH e a ISDF ainda não foram adaptadas no Brasil ou na Colômbia.

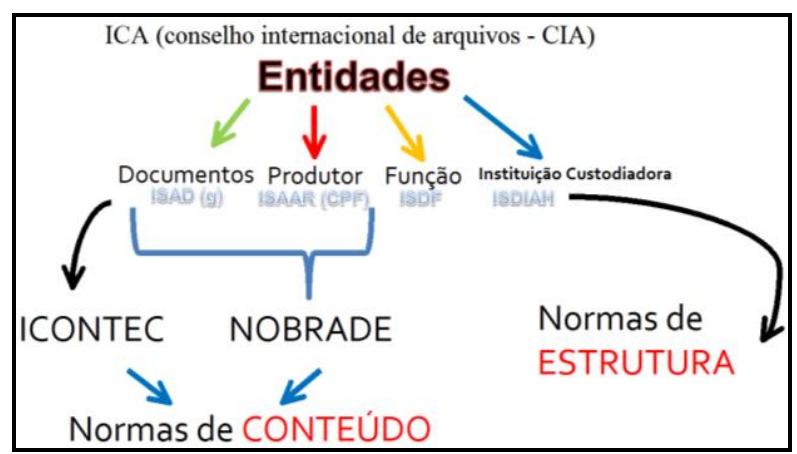

Figura 4: Adaptações das normas de descrição desenvolvidas pelo ICA no Brasil e na Colômbia.

A descrição, portanto, tem o intuito de atribuir significado ao objeto ou a informação e não apenas a função de ser um processo para construção de instrumentos de pesquisa. Porém, além disso, também é um importante aliado da interoperabilidade e de acesso, pois a descri- ção é uma representação de um documento ou de uma informação.

Na normalização da descrição encontram-se também elementos de significado nas etiquetas, como, por exemplo, o elemento descritivo "âmbito e conteúdo" este se refere a uma determinada informação. A norma apresenta junto ao elemento descritivo uma explicação do seu objetivo e as regras que ele exige para o preenchimento de cada campo descritivo. $\mathrm{O}$ objetivo do elemento âmbito e conteúdo é, segundo a norma, capacitar os usuários a avaliarem a potencial relevância da unidade de descrição. A regra para a aplicação deste elemento, por outro lado, é fornecer, de acordo com o nível de descrição, um sumário do âmbito (como período de tempo e localização geográfica) e do conteúdo (como formas dos documentos, assuntos, processos administrativos) da unidade de descrição" ( $\operatorname{Isad}(G)$, 2000, p. 30-31).

Assim, se o agente descritor seguir corretamente os passos da norma, estará, ao mesmo tempo, tornando a representação duplamente significativa. Isto é, de um lado atribui significado pelo ato de descrever, e de outro pelo uso da etiqueta normalizada, que terá o mesmo objetivo, regra e aplicação em qualquer lugar do mundo. Se a descrição é a representação de um objeto ou informação e tem função de dar significado ao objeto representado, e se juntamente o estabelecimento da norma atribui significado, esses conteúdos trazidos para o ambiente Web tornam-se um ambiente com a presença de significado mecânico e automático, pois a máquina terá o conhecimento do que se refere aquela informação.

A normalização ainda apresenta aspectos de compatibilidade, pois facilita o acesso à informação e à construção de suportes de descrição arquivística. Possibilita também o intercâmbio de informações, contribuindo então para a interoperabilidade de sistemas e para uso na Web.

\section{O AtoM como recurso descritivo para a interoperabilidade}

Para a efetividade da interoperabilidade é necessário que os ambientes Web sejam baseados em normas, interconectados, multilíngues e participativos. Esses quatro elementos estão presentes em um ambiente já existente: o software livre AtoM.

O AtoM é um software desenvolvido para a criação de um ambiente Web de descrição arquivística. O sistema foi inicialmente intitulado ICA-AtoM, pois provinha de uma parceria entre o Conselho Internacional de Arquivos (ICA) e o grupo Access to Memory (AtoM) que, posteriormente, deixou de existir, pois o ICA percebeu que ter o seu nome atrelado ao sistema poderia limitálo a instituições arquivísticas. Flores e Hedlund (2014) 
descreveram o software quando ele ainda era desenvolvido pela parceria:

o software ICA-AtoM (acrônimo para International Council Archives - Access to Memory) é resultante de um projeto de mesmo nome. O projeto ICA-AtoM teve seu início através de um relatório em 2003, da Comissão de Tecnologia da Informação do ICA, que estabelecia requisitos funcionais para um "Open Source Archival Resource Information System" (OSARIS) (tradução nossa: sistema aberto de pesquisa em informações arquivísticas). (p. 26).

Atualmente, o projeto AtoM está sob a gerência do grupo Access to Memory. No entanto, as versões produzidas em parceria entre o ICA e o grupo Access to Memory (AtoM) continuam disponíveis na Web e podem ser usadas normalmente, pois o sistema não mudou estruturalmente. Apesar da mudança no grupo de desenvolvimento, o trabalho com o sistema continua em curso e seus resultados são disponibilizados livremente na Web, podendo ser incorporadas todas as versões do sistema ICA-AtoM e AtoM, que coexistem e são interoperáveis. A nomenclatura também persiste, pois o ICA não retirou seu apoio aos desenvolvedores do sistema.

O AtoM tem sido bastante utilizado no Brasil por instituições de arquivo, como o Arquivo Nacional e o Arquivo Público de São Paulo, para disponibilizar as descrições dos documentos na Web devido principalmente à sua licença de software livre e sua gratuidade.

É necessário destacar que o sistema AtoM não é GED. Certamente ambos os sistemas computacionais de informação são necessários, embora diferentes. Enquanto os softwares de Gerenciamento Eletrônico de Documentos (GED) têm a função de gerenciar o trâmite documental dentro de uma instituição, o AtoM atua na descrição dos documentos, independentemente do uso corrente do documento dentro da instituição. Enquanto o GED atua na parte de controle de uso do documento, como um conjunto de tecnologias que permitem o gerenciamento dos documentos em forma digital, o AtoM atua no acesso à informação documental. Documentos podem provir dos mais diversos suportes, tais como papel, microfilme, imagem, som, ou mesmo arquivos já criados na forma digital (planilhas eletrônicas, arquivos de texto etc.). A preferência atual por formatos digitais justifica-se pela ineficiência e onerosidade dos acúmulos de documentos tradicionais em papel, tais como: custos com espaço de armazenamento, pessoal especializado, morosidade na recuperação e destruição precoce da informação.

Mesmo que ambos os sistemas lidem com o mesmo documento, o objetivo, a ênfase e o tratamento são diferentes. Enquanto o GED gerencia o trâmite e controla o acesso dos usuários do sistema, o AtoM descreve o documento para proporcionar o acesso e facilitar a recuperação da informação para os usuários. O GED sem uma interface de busca na Web, não representaria ne- nhuma garantia da atribuição de significado para a Web Semântica. O AtoM, ao contrário, é um sistema de descrição multinível e de representação da informação que também é multiarquivo e multilíngue, baseado em normas, um software livre - e, portanto, de código aberto (que possibilita a interoperabilidade) - e baseado em ambiente Web.

Para melhor entender as distinções, que têm sido motivo de dúvidas e incompreensão, apontam-se a seguir diferenças fundamentais que demonstram a necessidade da interoperabilidade entre as duas instâncias nos arquivos.

O GED é um processo como um todo, que trata os documentos originalmente eletrônicos e aqueles transformados em forma eletrônica através da digitalização, que realiza a transformação a partir de documentos dos mais variados meios físicos, tais como papel, microficha, filmes, áudio etc. A digitalização é, portanto, somente uma das etapas de um processo de GED. A diversidade fica clara quando, por exemplo, uma instituição conta com um sistema GED, um arquivo central e diversos departamentos e seções. Nela um departamento de materiais pode dar início a um Processo de Compra de Material de Escritório (PCME) e encaminhar ao departamento de finanças. Para que esse PCME chegue até o departamento de finanças ele deve passar pelo arquivo central. Com isso, o departamento de materiais envia o documento PCME ao arquivo central (AC). Ao receber o processo via correio interno, o AC pelo sistema GED acusa o recebimento do arquivo, despacha o PCME ao departamento de finanças que, ao recebê-lo, também acusa o recebimento pelo GED. Cada vez que um documento qualquer sair de uma seção/departamento e chegar a outro será registrado no sistema.

Nessa situação exemplar é possível perceber que o GED tem grande importância no mapeamento do trâmite do documental e no controle de acesso, garantindo a integralidade do documento, diminuindo as possibilidades de perda e/ou de qualquer dano ao documento.

Se, por outro lado, uma instituição pretende disponibilizar os documentos para a recuperação de qualquer usuário, o AtoM irá descrever a instituição, a sua função, os fundos e sub-fundos, os grupos e sub-grupos e quaisquer níveis de classificação, podendo chegar ao item documental que na situação paradigmática descrita acima, é o PCME. Nela, o AtoM por ser um sistema de descrição multinível para que no mesmo ambiente seja feita a descrição desde a instituição até o item documental.

Outro ponto importante nessa diferenciação entre os sistemas GED e AtoM, é que o AtoM é um software desenhado para Web, enquanto um sistema GED nem sempre funciona em ambiente Web. No caso de um sistema GED que não tenha interface, ele terá que ser 
adaptado. Caso não seja possível, a instituição terá que adquirir outro sistema que funcione em ambiente Web.

A ênfase do AtoM é, ao contrário, o acesso. O processo de descrição tem como fim dar significado ao objeto (ou informação) para facilitar o acesso à informação. A descrição é um tipo de representação da informação que visa recuperar o contexto histórico, fazer o registro da identificação e localização da peça ou documento dentro do acervo, e disponibilizar os metadados para pesquisa. Depois de realizadas as descrições do acervo é possível criar instrumentos de pesquisa como guias, catálogos, inventários e índices no AtoM. Cada instrumento de pesquisa é construído a partir de um nível de descrição e ao agrupar as descrições no nível de coleção, constrói-se simultaneamente o guia de coleções: ao agrupar descrições de todos os documentos do acervo constrói-se o inventário do acervo, etc.

No AtoM, o momento de criação de instrumento de pesquisa é automatizado de maneira multinível, como se preconiza a organização de arquivos, pois, como visto, nele são contempladas as descrições desde o nível mais abrangente - a instituição - até o mais específico - a unidade documental. Dessa forma, quando um usuário realiza sua busca de informação no AtoM, ele recupera no mesmo ambiente informações em todos os níveis de descrição. A descrição contempla campos para registro dos metadados e informação quanto ao acervo, à instituição, ao estado de conservação das peças, entre outros. Por meio dela também é possível recuperar a ligação entre os documentos do acervo, mesmo que estes estejam guardados em locais diferentes. Tais ligações entre os documentos se realizam nos campos de descrição destinados a pontos de acesso e a indexação e o acervo torna-se, assim, mais coeso. O AtoM é, portanto, um sistema criado para ambiente Web e está preparado para atuar também na interoperabilidade, onde ainda poderá contribuir no desenvolvimento desta Web na reflexão das formas de atribuição do significado.

\section{A descrição arquivística no Brasil e na Colômbia para o acesso público e seus desafios}

No Brasil, apesar das normas serem conhecidas, ainda existe um desafio a ser superado: a implementação destas normas e do sistema AtoM nos arquivos. Nos levantamentos preliminares realizados nesta investigação percebeu-se que poucos municípios brasileiros têm uma instituição de Arquivo.

Silva e Jorente (2013, 2014a, 2014b) e Dal'Evedove e Jorente $(2014,2015)$ desenvolveram pesquisa para mapear a disponibilização da informação nos Websites dos Arquivos Públicos Permanentes dos municípios Brasileiros com número igual ou maior a 100.000 habitantes. Encontrou-se que, nos 26 estados brasileiros pesquisados, o estado que mais possui Arquivos Municipais é o estado de São Paulo. Entretanto, dos dez mu- nicípios mais populosos do estado de São Paulo apenas seis possuem Arquivos e desses seis, apenas dois municípios fornecem algum tipo de acesso aos documentos de forma online via Web. Dos dois municípios que fornecem acesso, apenas o Arquivo Municipal da cidade de Campinas encontra-se descrito por meio do software AtoM. Ainda na esfera pública, sabe-se que o Arquivo Público do Estado de São Paulo também utiliza o AtoM. No Rio Grande do Sul, por outro lado, a Universidade Federal de Santa Maria - UFSM, também faz uso do software. Aponta-se que, na cidade de Marília, São Paulo, com cerca de 240.000 habitantes, inicia-se trabalho de capacitação de funcionários do Arquivo Público a partir de convênio em andamento entre a Prefeitura Municipal e a Universidade Estadual Paulista, para a disponibilização de documentos históricos da cidade em ambiente Web, no software AtoM, sob a orientação do grupo responsável pelo Laboratório de Pesquisa em Design e Recuperação da Informação LADRI.

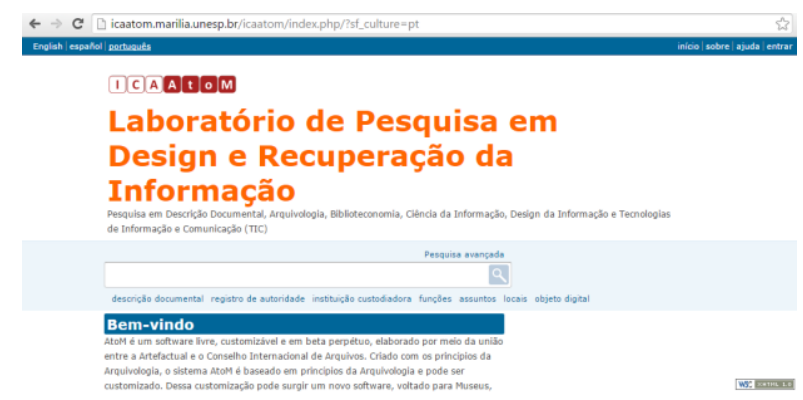

Figura 5: Página de entrada do site do LADRI com o sistema AtoM. Disponível em: <icaatom.marilia.unesp.br>.

Destaca-se que este trabalho de pesquisa ação pretende a criação de um modelo de aplicação para ser replicado no contexto de outros municípios do Estado. A importância da iniciativa deve-se à necessidade criada pela mudança de cenário nas políticas públicas de acesso à informação diante da publicação da Lei 12.527/11 conhecida como "Lei de Acesso a Informação" - LAI. O incentivo às instituições públicas para aplicar as normas de descrição e o AtoM em seus arquivos, reside na gratuidade e resposta efetiva das determinações da LAI.

No caso da Colômbia, o Arquivo Geral da Nação da Colômbia (AGN) conta na atualidade (2016) com um Grupo de Inovação e Apropriação de Tecnologias da Informação Arquivística que tem vinculado dentro de suas frentes de trabalho, o Laboratório de Inovação Digital Arquivística (LIDA), que desenvolve projetos de inovação mediante a criação de conteúdos digitais, educativos e sem custo, baseados em software livre, processos e novas tecnologias. Eles não sugerem nenhuma solução tecnológica ou software aos arquivos colombianos, mas estão promovendo uma valorização de ferramentas e aplicações de código aberto na área da descrição documental, como é o caso do AtoM (Access to Memory), para o qual foi elaborado um manual de 
usuário e um manual de instalação, ambos disponibilizados no site do LIDA (1).

Os manuais foram criados para facilitar o uso e a familiarização com o AtoM versão 2.0.1, nas entidades públicas e particulares que cumprem funções de interesse público. Entretanto, embora tenham-se criado os manuais, não há controle nem mensuração do número das instituições colombianas que utilizam o AtoM. Por este motivo não é possível mensurar o uso do software no país, embora tenham sido realizadas pesquisas com o envio de formulários para instituições colombianas de destaque, com respostas negativas quanto a implantação do AtoM, mesmo que este seja conhecido por algumas delas.

Nesta pesquisa, realizada em alguns arquivos públicos da Colômbia pode-se, entretanto, observar que poucos conhecem a existência do AtoM e que até o momento não se tem notícia de sua aplicação. Algumas instituições fazem uso de softwares proprietários como Mercurio Sistema de Gestión Documental e ENKI JANIUM derivado do Gestor Bibliográfico JANIUM. Assim, pesquisas sobre a descrição documental e a implementação do AtoM na Colômbia, tornam-se necessárias e imprescindíveis para apoiar os avanços arquivísticos, a normalização dos conteúdos e o acesso efetivo dos sujeitos que buscam informação.

A pesquisa irá contribuir no desenvolvimento desta Web na reflexão das formas de atribuição do significado.

\section{Considerações finais}

O perfil dos indivíduos que buscam informações em instituições de informação e na Web tem passado por mudanças, pois estes desejam informações melhores e completas, buscas mais qualificadas às suas necessidades de informações; desejam ter acesso aos documentos de arquivo (fonte primária de informação) e demonstram uma postura proativa na recuperação da informação na Web.

A descrição arquivística, a normalização da descrição, ambientes colaborativos e ambientes interoperáveis, podem ser alternativas satisfatórias a estas demandas, desde as questões de normas e padrões até a proposta do sistema AtoM, como resposta ao acesso e a interoperabilidade. Afinal, o objetivo de uma descrição arquivística é proporcionar o acesso à representação dos documentos de arquivo por meio de instrumentos de pesquisa e o uso do AtoM. Com este acesso, desencadeia-se processos de visibilidade que levam à compreensão da necessidade de preservação dos arquivos públicos, ou não.

\section{Notas}

(1) Site do Laboratório de Inovação Digital Arquivística (LIDA). Disponível em: <http://lida.archivogeneral.gov. co/> Acesso em: 14 jan. 2015.

\section{Referências}

Arquivo Nacional - Brasil (2004). Conselho nacional de arquivos. Dicionário Brasileiro de Terminologia Arquivística. Rio de janeiro: arquivo nacional, 2004.

Carpes, F. S.; Flores, D. (2014). Instrumento de descrição arquivística em meio eletrônico: definição do quadro de padrões, normas e metadados. // Perspectivas em Ciência da Informação 19:4 (2014) 67-80.

Dal' Evedove S. R.; Jorente, M. J. V. (2014). A disponibilização da Informação em Websites de Arquivos Públicos Permanentes da Região Sul do Brasil. // Relatório Final de Atividades 2014. Programa de Iniciação Científica (PIBIC) CNPq, 2014.

Dal' Evedove S. R.; Jorente, M. J. V. (2015). A disponibilização da Informação em Websites de Arquivos Públicos Permanentes da Região Centro-oeste do Brasil. // Relatório Parcial de Atividades 2015. Programa de Iniciação Científica (PIBIC) CNPq, 2015.

Flores, D.; Hedlund, D. C. (2014). A Preservação do Patrimônio Documental através da Produção de Instrumentos de Pesquisa Arquivísticos e da Implementação de Repositórios Arquivísticos Digitais. // Série Patrimônio Cultural e Extensão Universitária, 3 (2014). Acesso em http://www.iphan.gov.br/baixaFcdAnexo.do?id=4324.

Icontec. (1997). Norma Técnica Colombiana 4095: Norma General para la descripción archivística. // Bogotá: Icontec, 1997.

ISAD (G) (2000). Norma Geral Internacional de Descrição arquivíctica - Segunda Edição. // Adotada pelo Comitê de Normas de Descrição, Estocolmo, Suécia, 1999. Rio de Janeiro, 2000. Acesso em http://www.ica.org/en/isadg-general-internationalstandard-archival-description-second-edition.

Llanes Padrón, D. (2011). La Representación normalizada de los documentos. Estudio comparado de normas de descripción archivística. // Salamanca: Universidad de Salamanca. Tese. 2011.

Silva, A. R.; Jorente, M. J. V. (2013). A disponibilização da Informação em Websites de Arquivos Públicos Permanentes da Região Sudeste do Brasil. // Relatório Parcial de Atividades 2013. Programa de Iniciação Científica (PIBIC) CNPq, 2013.

Silva, T. C.; Jorente, M. J. V. (2014a) A disponibilização da Informação em Websites de Arquivos Públicos Permanentes da Região Sudeste do Brasil. // Relatório Final de Atividades 2014. Programa de Iniciação Científica (PIBIC) CNPq, 2014.

Silva, T. C.; Jorente, M. J. V. (2014b). A disponibilização da Informação em Websites de Arquivos Públicos Permanentes da Região Nordeste do Brasil. // Relatório Parcial de Atividades 2014. Programa de Iniciação Científica (PIBIC) CNPq, 2014.

Copyright: (C) 2016 Jorente et al. This is an open-access article distributed under the terms of the Creative Commons CC Attribution-ShareAlike (CC BY-SA), which permits use, distribution, and reproduction in any medium, under the identical terms, and provided the original author and source are credited. 